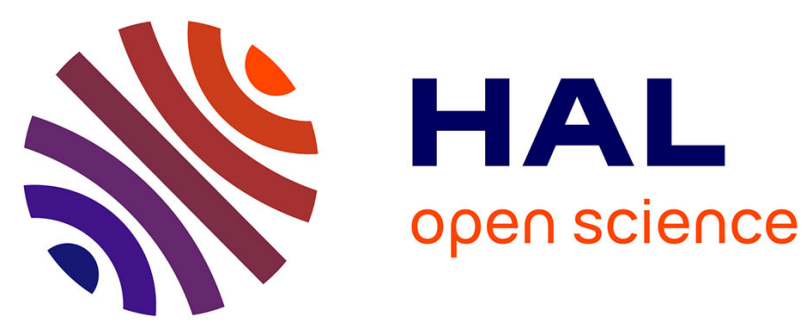

\title{
EQUIVOX: an example of adaptation using an artificial neural network on a case-based reasoning platform
}

\author{
Julien Henriet, Brigitte Chebel-Morello, Michel Salomon, Jad Farah, Rémy \\ Laurent, Marc Sauget, David Broggio, Didier Franck, Libor Makovicka
}

\section{- To cite this version:}

Julien Henriet, Brigitte Chebel-Morello, Michel Salomon, Jad Farah, Rémy Laurent, et al.. EQUIVOX: an example of adaptation using an artificial neural network on a case-based reasoning platform. Biomedical Engineering: Applications, Basis and Communications, 2013, 25, 1350027: 13 p. 10.4015/S1016237213500270 . hal-00956233

\section{HAL Id: hal-00956233 \\ https://hal.science/hal-00956233}

Submitted on 6 Mar 2014

HAL is a multi-disciplinary open access archive for the deposit and dissemination of scientific research documents, whether they are published or not. The documents may come from teaching and research institutions in France or abroad, or from public or private research centers.
L'archive ouverte pluridisciplinaire HAL, est destinée au dépôt et à la diffusion de documents scientifiques de niveau recherche, publiés ou non, émanant des établissements d'enseignement et de recherche français ou étrangers, des laboratoires publics ou privés. 


\title{
EQUIVOX: AN EXAMPLE OF ADAPTATION USING AN ARTIFICIAL NEURAL NETWORK ON A CASE-BASED REASONING PLATFORM
}

\author{
J. Henriet ${ }^{1}$, B. Chebel-Morello ${ }^{2}$, M. Salomon ${ }^{3}$, J. Farah ${ }^{4}$, R. Laurent ${ }^{1}$, M. Sauget ${ }^{1}$, D. Broggio ${ }^{4}$, D. \\ Franck $^{4}$, L. Makovicka ${ }^{1}$ \\ ${ }^{1}$ Université de Franche-Comté, IRMA/ENISYS/FEMTO-ST UMR 6174 CNRS, 4 Place Tharradin, \\ 25200 Montbéliard, France \\ ${ }^{2}$ Université de Franche-Comté, COSMI/AS2M/FEMTO-ST UMR 6174 CNRS, 24 Rue Alain Savary, \\ 25000 Besançon, France \\ ${ }^{3}$ Université de Franche-Comté, AND/LIFC, Rue Engel Gros, 90000 Belfort, France \\ ${ }^{4}$ French Institute of Radiological Protection and Nuclear Safety, Internal Dose Assessment \\ Laboratory, DRPH/SDI/LEDI, BP-17 F-92262 Fontenay-aux-Roses, France
}

\begin{abstract}
In case of a radiological emergency situation involving accidental human exposure, a dosimetry evaluation must be established as soon as possible. In most cases, this evaluation is based on numerical representations and models of victims. Unfortunately, personalised and realistic human representations are often unavailable for the exposed subjects. However, accuracy of treatment depends on the similarity of the phantom to the victim. The EquiVox platform (Research of Equivalent Voxel phantom) developed in this study uses Case-Based Reasoning (CBR) principles to retrieve and adapt, from among a set of existing phantoms, the one to represent the victim. This paper introduces the EquiVox platform and the Artificial Neural Network (ANN) developed to interpolate the victim's 3D lung contours. The results obtained for the choice and construction of the contours are presented and discussed.
\end{abstract}

\section{KEY WORDS}

Case-Based Reasoning (CBR), Adaptation, Artificial Neural Network (ANN), 3D personalised phantoms, radiation protection.

\section{INTRODUCTION}

In case of accidental exposure to radiation, a dosimetry evaluation must be established for each victim as soon as possible. In most cases, this evaluation is based on available 3D voxel Phantoms, numerical models created from medical images to represent the imaged subject with maximum realism. Examples of voxel phantoms for dosimetric assessment following internal contamination or external exposure can be found $[1,2,3,4,5]$. However, even when medical images are available, the victim's specific phantom is not always accessible since its construction is delicate and time consuming, and in emergency cases such time and effort are unaffordable. Moreover, medical images are avoided so as to prevent any additional exposure to radiation. Thus, existing models are used even if their characteristics differ from the victim's biometrical data. Dosimetry assessment accuracy and the resulting decontaminating medical action is nevertheless highly dependent on the similarity between phantom and victim. Hence, the actual work aims at assisting the physician in choosing the fittest phantom from the existing and available ones.

Case-Based Reasoning (CBR) is a problem solving method that uses similar solutions from similar past problems in order to solve new problems [6]. Based on the ReEPh (Research of Equivalent Phantom) project $[7,8]$, the EquiVox project strikes a new path in the field of problem solving methods 
for the retrieval and the adaptation of 3D phantoms. It uses the CBR-approach to find the most similar phantom(s) within any set of phantoms and then attempts to adapt them to the characteristics of the target case (the victim). Actually, $\mathrm{ReEPh}$ was not able to automatically create new phantoms: it only allowed the retrieval and the classification of the stored phantoms through the Graphical User Interface (GUI) and the implementation of a K-nearer neighbour (K-nn) algorithm. Whereas the ReEPh project's main purposes were only to retrieve the most accurate stored phantom(s), considering a set of known characteristics, and to help the experts in their choice, EquiVox introduces an original tool allowing the stored phantoms to be adapted to the victim. This automatic adaptation uses an Artifical Neural Network (ANN) tool $[9,10,11]$ and requires implementation of a learning step before the CBR-adaptation process.

A large number of phantoms can be found in the literature $[12,13,14,15,16,17,18]$, and radiation protection is also divided into numerous sub-domains. Indeed, some phantoms are commonly used by experts for external radiotherapy, and others are used by other physicians for evaluation of internal doses received. In fact, each expert has his own set of 10 to 20 phantoms. When physician's usual phantoms are all too distant from the victim, the expert must create a new one. Indeed, using iterative 3D dilatations and contractions, physicians modify the contours of the 3D organs of their phantoms until they correspond to those of the victim. They then put them together and obtain the final phantom on which the computations will be based [19]. Thus, the adaptation rules are guided by their experience and knowledge. The main challenge of EquiVox is to reproduce the same transformation process automatically, without human intervention.

Another requirement of ReEPh and EquiVox is to be able to use any set of phantoms and to help the physician to capitalise on them. We also hope that such a platform will be used to automatically create a well-fitting phantom for each victim in order to increase the accuracy of dose calculations. At this step of the implementation of EquiVox, we relied on phantoms usually used by a team of experts for pulmonary anthroporadiametry which consists of evaluating the internal dose inhaled.

\section{CASE MODELISATION}

When radiation overexposure occurs, a dosimetric report must be established for all victims. For each victim, the experts' first task is to choose the most accurate 3D phantom considering the information known about the victim. Each phantom has its own characteristics and is chosen by comparing the victim's available measurements and information to his/her characteristics. The phantom is thus chosen by analogy.

As explained, the experts choose the phantom according to the characteristics of the victim. We have exhausted the list of useful characteristics furnished by the physicians of the French Institute of Radiation and Protection (IRSN).

Thus, in EquiVox, a problem is described as a set of $r$ descriptors $\left\{d_{l}, \ldots, d_{r}\right\}$.

Each expert has his own set of $n$ phantoms: $S P=\left\{P_{l}, \ldots, P_{n}\right\}$.

Each $P_{i}$ is the solution part of a case and represents the contours of $m$ organs.

Each organ $O$ is a set of $q$ points joined by a Delaunay mesh [20]: $P_{i}=\left\{P_{i}{ }^{l}, \ldots, P_{i}^{m}\right\}$ and $P_{i}^{o}=\left\{C_{l}^{i, o}, \ldots\right.$, $\left.C_{q}^{i, o}\right\}$ where $C_{j}^{i, o}$ denotes the 3D coordinates of point $j$ of organ $O$ of phantom $P_{i} . O \in$ \{lung, heart, liver, sternum, ribs, scapulae, spine, breasts, skin, oesophagus and thorax 3 .

Finally, a case $i$ is: $i=\left\{\left\{d_{l}^{i}, \ldots, d_{r}^{i}\right\}, P_{i}\right\}$. We will note $t$ as target case. 


\section{RETRIEVAL PHASE}

The purpose of this phase is to sort the phantoms of the EquiVox case-base according to information concerning the victim, even if incomplete. Hence, the number of known descriptors influences the level of confidence in the proposed EquiVox ranking. Thus, along with the similarity index $\left(S_{i}\right)$, a confidence index $(C)$ is assessed to associate an error with the retrieved solution.

In addition, some descriptors may be very important for some types of calculations while others may be totally neglected. Since the purpose of EquiVox is to retrieve and adapt phantoms, whatever their use, our platform must take into account the importance of each descriptor. Thus, the descriptors were weighted, taking into account their importance and influence. As presented in Equations (1) and (2), these weights $\left\{\lambda_{1}, \ldots, \lambda_{r}\right\}$ are quantitative values associated to each descriptor, amplifying or reducing the differences between $t$ and $i$. They thus stress on the relative influence that one measure represents in comparison to the others.

In fact, when a new problem occurs, some of the victim's characteristics may be unavailable. Thus, a Boolean value $\delta_{k}$ is associated to each $d_{k}$. $\delta_{k}$ is equal to 0 if the value of $d_{k}$ is unknown, and to 1 otherwise.

Hence, a classical algorithm for similarity calculation was used, namely the K-nn Algorithm that enables a weight to be applied to the descriptor values.

The $S_{i}$ value is equivalent to the sum of the distances between the descriptors of $i$ and $t$, each weighted accordingly. It is given by the following equation:

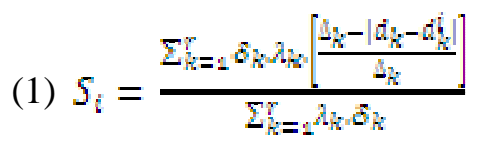

$\Delta_{k}$ is the difference between the maximum and the minimum known values that the descriptor $d_{k}$ can take. The $S_{i}$ value is always between 0 and 1 . The greater the similarity of $i$ to $t$, the closer the $S_{i}$ value is to 1 .

Since $S_{i}$ only takes into account the known values of $t$, the confidence index $C$ must be taken into account to define the calculation uncertainty. The more values we know, the higher the confidence index. Indeed, if the victim's age is the only known criteria, the similarity value calculated is totally insignificant. So $C$ takes into account the number of known values according to the following formula:

$$
\text { (1) } C=\frac{\sum_{k=2}^{r} \varepsilon_{k} d_{k}}{\sum_{k=2}^{r} d_{k}}
$$

In addition, the EquiVox GUI (Graphical User Interface) allows users to graphically compare $t$ to each source case $i$ of the case-base. The plotted graph represents a multitude of axes, each equivalent to one descriptor of the case. On each axis, the value of the target case descriptor and the one of the selected source case are reported. When no descriptor value is given for $t$, the axis value is put at null; the descriptor value of $i$ is still reported on the graph. If the characteristics of $i$ and $t$ are the same, the areas will be merged. Thus, the larger the overlapping areas, the more similar $i$ is to $t$.

\section{ADAPTATION OF 3D LUNG CONTOURS}

Once a matching case is retrieved, the expert can decide either to use the phantom of the most similar source cases, or require the EquiVox platform to generate a new phantom, adapting the source cases to 
the target one. Indeed, if some available phantom measurements are too different from those of the victim, the expert may decide to adapt one of them or even to create a new phantom which may be reused for other problems later. Thus, when the expert requires the generation of a new phantom, the contours of the $m$ organs are expected.

Actually, the first organs experts create in such a personalised process are the lungs. The positions and volumes of the other organs are deduced from the lungs. Thus, we first considered the adaptation of $3 \mathrm{D}$ lung contours.

\subsection{Solution space modelisation for 3D lung contours}

As presented in Section 2, the lung contours of phantom $P_{i}$ are defined in 3D by a set of $q$ points joined by a Delaunay mesh: $P_{i}^{\text {lung }}=\left\{C_{l}^{i \text {, llung }}, \ldots, C_{q}^{\text {i,lung }}\right\}$ where $C_{k}^{\text {i, lung }}$ denotes the $3 \mathrm{D}$ coordinates of point $k$ : $C_{k}^{i, \text { lung }}=\left\{x_{k}^{i, \text { lung }}, y_{k}^{i, \text { lung }}, z_{k}^{i, \text { lung }}\right\}$.

For all the phantoms, the same number of points defines the 3D contours of the lungs. The points have been plotted in the same order and in the same Cartesian coordinate system. Thus, the task of the lung contour-adaptation phase of EquiVox consists of interpolating the 3D coordinates of the points of $t$ in the same order and in the same Cartesian coordinate system. A Delaunay mesh can then be applied so as create the contours of the lungs of $t$.

\subsection{Adaptation rules}

Actually, lung contours and volumes depend mostly on the size of the victim. Indeed, for the lungs, I. Clairand et al. [21] proved that the height of a person prevailed for their geometry and volume.

Thus, when experts decide to create the lung contours of a victim, they choose the lung contours of the stored phantom whose height is the closest without taking into account any other characteristic. The adaptations are usually done manually, applying mathematical transformations (2D and 3D contractions and dilations [19]). These transformations are carried out through 3D modelling tools (such as Rhinoceros [22] or CATIA [23]).

In addition, these transformations are only driven by experience, trials and errors, and may take many hours or more. The delay also increases with the number of victims whereas the problem resolution delay may be limited. Indeed, in the case of massive irradiation for example, when a disaster such as a nuclear explosion occurs, dosimetric reports are required for hundreds of people of different sizes.

In fact, the creation of new lung contours requires a fast data-driven method, and since there is no physical law to governing its design, the expert is not able to explicit a rule for the transformation of the lung contours.

\subsection{Method}

Since the mesh and the number of points are not variable, the adaptation must be carried out on the point coordinates of the lung contours, point by point. Since no formal equation exists, we must discover through a learning method the rules that transform the coordinates of the points on one lung contour into other coordinates.

Consequently, data-driven methods using inductive reasoning are the most suitable approaches; ANN and Fuzzy-ANN respond to these requirements. We chose ANN as the tool for this step, assuming this could serve as the basis for further work with Fuzzy-ANN if the first results were not convincing. 
We explored the possibility of using a multi-layer perceptron trained with a backpropagation-based method. Other interpolation methods were tested: polynomial and Spline ones (cf Subsection 4.3.2).

\subsubsection{ANN inputs, outputs and topology}

To interpolate the 3D lung contours, the height is required. Actually, this is one of the descriptors of the EquiVox target and source cases. Let us note $h_{i}$ the descriptor corresponding to the height of the case $i$ and $h_{t}$, the height of the target case $t$.

Actually, two different configurations were tested: firstly one ANN with 9 inputs and 3 outputs, and a second one with 5 inputs and 1 output. For both of these configurations, two phantoms were considered:

- The source case inf for which $h_{i n f}$ is inferior and the closest to $h_{t}$;

- The source case sup for which $h_{\text {sup }}$ is superior and the closest to $h_{t}$.

For the first configuration, the trained ANN interpolates the 3 coordinates of each point of the lung contours separately. Thus, the 9 inputs permitting interpolation of the coordinates $C_{k}^{\text {lung }}$ of the point $k$ of $t$ are:

- The 3 coordinates of point $k$ of the lung contours of inf: $C_{k}^{\text {inf,lung }}=\left\{x_{k}^{\text {inf,lung }}, y_{k}^{\text {inf,lung }}, z_{k}^{\text {inf, lung }}\right\}$;

- The height of inf: $h_{\text {inf }}$;

- The 3 coordinates of point $k$ of the lung contours of sup: $C_{k}^{\text {sup,lung }}=\left\{x_{k}^{\text {sup,lung }}, y_{k}^{\text {sup,lung }}\right.$, $z_{k}^{\text {sup,lung }}$;

- The height of sup: $h_{\text {sup }}$;

- The height of the target case: $h_{t}$.

For the second configuration, 3 trained ANN are required: the first ANN learns how to interpolate first coordinate $x$, the second deals with $y$ and the third is dedicated to the interpolation of $z$. In fact, the same ANN is duplicated 3 times. The coordinates are learned separately, thus the inputs of the first ANN allowing the interpolation of coordinate $x_{k}$ from $t$ are:

- $\quad x_{k}^{\text {infllung. }}$

- $h_{\text {inf }}$;

- $x_{k}^{\text {sup,lung }}$

- $h_{\text {sup }}$;

- $h_{t}$.

For the second ANN $x_{k}^{\text {inf,lung }}$ and $x_{k}^{\text {sup,lung }}$ are respectively replaced by $y_{k}^{\text {inf,lung }}$ and $y_{k}^{\text {sup,lung }}$, and $z_{k}^{\text {inf,lung }}$ and $z_{k}^{\text {sup,lung }}$ for the third ANN.

The designed ANN are perceptrons having one hidden layer. There are 10 neurons on the hidden layer with a sigmoid activation function. The activation function of the neurons belonging to the output layer is linear.

Such topologies were also chosen and tested with success on the NEMOSIS platform [10] for a similar issue: considering a point inside the lungs of a patient, its initial position (when inspiration is maximum) and its final one (when inspiration is minimum), a similar ANN interpolated the positions of the point during an entire breathing cycle with an error inferior to the spatial resolution of the medical images on which the point had been plotted. For NEMOSIS, the number of neurons on the hidden layer was optimized using a validation set in addition to the learning set used for the learning 
step. Due to the small number of phantoms (12 phantoms), we decided, for this first step of the implementation of the adaptation, not to consider a validation set. Thus, the learning set was composed of 9 phantoms, while the 3 remaining phantoms belonged to the test set. We assumed that such a topology would deliver sufficiently accurate results. Nevertheless, such a strategy to optimise the number of neurons on the hidden layer would have to be implemented in later work.

\subsubsection{ANN learning set and training step}

Training ends when the difference between the expected and the obtained values is minimised. W. Hsieh [24] distinguished four algorithms based on the backpropagation method:

- The BFGS method (Broyden-Fletcher-Goldfarb-Shanno) is a quasi-Newton method, which approximates the value of the Hessian matrix of the second derivatives of the function to be minimised;

- The L-BFGS method (Limited memory - BFGS) is an adaptation of the BFGS method which optimises the computational resources to use. Both of these methods must be coupled with a Wolfe linear search in order to determine an optimal step size between two iterations;

- The Rprop (Resistant backpropagation) method proposes a first order algorithm but its complexity increases linearly with network topology;

- The iRpropPlus method is one of the fastest and also one of the most accurate algorithms [25]. This evolution of the Rprop method allows cancelling some synaptic weight updates in the neural network if a negative effect is observed.

All of these methods were implemented and tested in the EquiVox adaptation phase of lung contours. Different required precisions were also tested. Table 1 shows the algorithm that gives the best interpolations. 12 interpolations were tested and BFGS gave the best results 11 times. Thus, BFGS algorithm with 10-6 as required precision was chosen to train an ANN.

\section{IMPLEMENTATION, TESTS AND RESULTS}

\subsection{EquiVox architecture}

The Equivox platform has been implemented and tested on a Personal Computer equipped with an Intel Core 2 Duo CPU, $2.26 \mathrm{GHz}$, and $2 \mathrm{GiB}$ RAM. The source case descriptors are stored in a mySQL database management system (DBMS). Two programming languages were used: Java and C. The retrieval phase, the GUIs, and the storage phase modules developed by our team in Java call C programs also developped by our team for the adaptation phase. The phantoms were drawn using Rhino3D.

Figure 1 presents the technologies that were used and the data flows over the EquiVox architecture. All the phantoms are stored in Rhino3D files. Their characteristics are stored in a database (data flow $\# 0$ in Figure 1), the lung contours are extracted (data flow \#1) and then transmitted to the ANN training module (data flow \#2) which creates the ANN (data flow \#3). When a new phantom is required, the target case description is transmitted to the retrieval module (data flow \#4) which determines the similitude and confidence indices taking into account the source case (data flow \#5). If required by the experts, the lung adaptation module sends the characteristics of the source cases (data flow \#6) to the ANN interpolation module (data flow \#7) which loads the trained ANN (data flow \#8) and the coordinates of the contour of the lungs in question (data flow \#9) in order to create interpolated contours suited to the target case (data flow \#10).

\subsection{EquiVox test case-base}


The particular domain of pulmonary anthroporadiametry applied to female subjects was considered for the performance evaluation of the EquiVox retrieval and adaptation phase.

The EquiVox case-base used for the tests contained 24 whole 3D phantoms with 3D organ contours and characteristics. These phantoms were manually designed from the ICRP standard female phantom [12] for pulmonary anthroporadiametry computations by the team of internal dose evaluation of IRSN [19]. These 3D phantoms were developed to cover as well as possible the diversity in the female population: thoracic phantoms of cup sizes ranging from $\mathrm{A}$ to $\mathrm{F}$ and chest girth from 85 to 120 (European Standard Clothing Units) [26]. These phantoms were developed for in vivo lung counting optimisation where volume and weight precisions are available for the following structures: lungs, heart, liver, sternum, ribs, scapulae, spine, breasts, skin, and thorax. The following external measurements are also available: age, sex, height, weight, cup size, and chest girth (chest and underbust circumferences). Thus, all these female phantoms and characteristics formed the 24 source cases of the tested EquiVox case-base.

The experts determined a list of 14 descriptors having varying degrees of influence in the choice of phantom for this type of calculation. These descriptors are age, height, weight, sex, wether the victim smokes or not, thorax volume, lung volume, extrathoracic thickness, fat-muscle proportion, under-bust circumference, wrist diameter, chest circumference, heart volume, and the victim's origin (target case) / phantom (source case).

For the adaptation phase tests, the 3D lung contours of these 24 phantoms had been considered and extracted. In fact, there are 9 distinct 3D lung contours $L C_{i}, i \in\{1, \ldots, 9\}$. For example, a phantom with a 90B thorax and one with a 90C have the same 3D lung contours since breast and lung volumes and contours are not correlated at all. Thus, 9 distinct phantom heights are reported in Table 2. Each one of these 9 3D lung contours is composed of 26723 points plotted in the same order and in the same Cartesian reference system as required by EquiVox modelisation.

\subsection{Retrieval phase performances}

In order to evaluate the performance of the EquiVox retrieval phase, the measurements of 80 different female subjects randomly selected from the CAESAR database [27] (Civilian American and European Surface Anthropometry Resource database) were considered as target cases descriptions. The latter is a database of over 2000 optical scans of Italian and Danish male and female subjects. Some of their measurements (age, sex, origin, and weight) are also stored with these scans and the spatial resolution enabling calculation of chest girth, cup size, and the height of each subject.

Farah et al. $[19,28]$ determined 5 sets of victim characteristics which influence the pulmonary anthroporadiametry dose computations. The weights $\lambda_{k}$ of the associated descriptors from the set influencing the phantom choice the most were set at 4 , whereas the weights of those with no influence on that type of computation were set at 0 . In the case of in vivo counting, it is known that the chest circumference and lung volumes are the most important parameters [29]. Hence, their associated weights were given the highest value: 4 . Moreover, in this example, the weights associated to the internal volumes were set at 0 .

For each target case, we compared the source case the expert would have chosen to the classification proposed by the EquiVox retrieval phase. For 75 target cases, the experts and the EquiVox retrieval phase chose the same source case first. Thus, 5 times, the EquiVox retrieval phase put the source case chosen by the experts in second place. Consequently, in $93.75 \%$ of the cases, EquiVox chose the most accurate source case regarding the target case description. The 5 target cases, for which the EquiVox 
retrieval phase missed the most accurate solution, can be explained by the influence of all other informed descriptors (age, height, weight, etc.). In fact, the difference between the values of these descriptors in these 5 target cases adds up and leads to a low similarity index. In addition, when no descriptor weighting was assigned $\left(\lambda_{k}=1 \forall k=1, \ldots, 14\right)$, the EquiVox retrieval phase put the most accurate source case in first place only 54 times.

Table 3 presents an example of the similitude and confidence indexes obtained when comparing the same target case to two very different source cases. The descriptor values of source cases $85 \mathrm{~A}$ and $120 F$, and one of the tested target cases are presented. The weights are also reported in this table. The $S_{85 \mathrm{~A}}$ computed for source case $85 \mathrm{~A}$ was equal to 0.765 , meaning that the target case and $85 \mathrm{~A}$ corresponded up to $76.5 \%$ considering the available descriptor values. In the other column of Table 3, $S_{120 F}=0.573$ for that same target case. For that target case, $C=0.8$. In addition, when all the weights were set at $1, S_{85 A}=0.510, S_{120 F}=0.545$ and $C=0.622$. This example emphasizes the importance of the confidence index and also the weights associated to the descriptors: different configurations must be carefully studied by the experts before launching the EquiVox retrieval phase.

\subsection{EquiVox graph comparison module}

In addition to the classification, the EquiVox GUI allows the experts to graphically compare each source case to the target case. For example, Figure 2 shows the graphical representations of the comparisons of the Table 3 target case (dark areas of both graphs) to source cases $85 \mathrm{~A}$ and $120 \mathrm{~F}$ (light areas of both of the graphs). On each axis the values of one descriptor are plotted (that of the target case and that of the source case) taking into account the associated weight. Actually, for each descriptor, the weighted distance between the values is normalised according to the weights. For example, considering notations of Equations (1) and (2), the reported values on the axis corresponding

to $d_{k}$ would be $\frac{A_{k} d d_{k}}{D_{k}}$ assuming $D_{k}$ is the maximum possible value for this descriptor. Moreover, the axes on which the victim values are known are dynamically brought together in the same area of the graph. Nevertheless, it is also possible for the expert to visualise the graph without normalisation and/or bringing them together.

The merged areas represent the similarities between the two cases. Considering $85 \mathrm{~A}$, the known characteristics of the victim match reasonably well, and thus most of the points on the different axes are merged, whereas they are very different on the graph comparing the same target case to $120 \mathrm{~F}$. With this GUI, the similarity of cases can be straightforwardly observed and the observation is confirmed by the numerical values obtained $\left(S_{85 A}\right.$ and $\left.S_{120 F}\right)$. It is also very simple to see what the critical characteristics are and to form an idea of the differences between the two cases. In our case, some descriptors, such as sex or origin, which may be considered as the most important criteria, are of limited influence for the $S_{i}$ calculation. Indeed, all the source cases and the tested target cases are of the same sex and origin (European female).

\subsection{Performance of the 3D lung contour adaptation}

The method was validated in three steps. First, different interpolation methods were tested over a sample of 10 points on each set of 3D lung contours. Then, a second round of tests was performed on all the sets of lung contour coordinates in order to determine the best ANN configuration and topology. Finally, we evaluated the performance of the EquiVox adaptation strategy using 3 new 3D lung contours.

\subsubsection{Interpolation method choice}


The best ANN configuration has been compared to a polynomial (Newton, of degree 2) and a Spline interpolation method. The Newton and Spline interpolators were implemented with Scilab 5.3.2 [30]. The Newton interpolation function proposed by J. Ponce and R. Brette in [31] and the Spline method proposed by Scilab were implemented and adapted. The coordinates of 10 points were randomly extracted from all the lung contours. For each method, a cross-validation for the same 10 points was undertaken using the same 9 lung contours of Table 2. Figure 3 presents the mean distances between interpolated and expected coordinates. This figure shows that the polynomial interpolation produced the greatest errors among the three tested interpolations. A factor nearly equal to 10 can be observed between the polynomial interpolation and that of the Spline or the ANN. The Spline and the ANN interpolations gave closer errors. Nevertheless, for all the tested cases, the ANN interpolation errors were inferior to the Spline ones 6 times and equal only once. These results prove the superiority of the ANN interpolations over the other methods since the ANN interpolation gave a more accurate result in all the tested cases.

\subsubsection{ANN configuration choice}

At this step of the choice, a cross-validation was performed since the learning set contained only 9 different sets of coordinates (3D lung contours $L C$ ): $L S=\left\{L C_{i}\right\}, i \in\{1, \ldots, 9\}$. Seven different subsets of $L S$ were considered and tested. The subset $L S_{j}$ was built to satisfy $L S_{j}=\left\{L C_{i}\right\}, i \in\{1, \ldots, 9\}$ and $i \neq$ $j \forall j \in\{2, \ldots, 8\}$. Indeed $j$ was never fixed at 1 or 9 since a smaller and higher phantom are always required for the interpolation (cf. Subsection 4.3.1). When the ANN was trained with $L S_{j}$, it was evaluated with the interpolation of $L C_{j}$. The configurations with 9 and 5 inputs were evaluated (cf Subsection 4.3.1). Since the number of learning sets is not important, we also explored the possibility of using the median value instead of the mean-square error to stop the ANN learning. Indeed, the median value converges more rapidly than that of the mean-square to the "average" value of a small set of items. We assumed this property of the median could influence the accuracy of the ANN interpolations.

Table 4 presents mean errors obtained when the different trained ANN (cf. subsection 4.3.1) interpolated each $L C_{i}, I \in\{2, \ldots, 8\}$. For the configuration with xyz interpolated together, and mean square error as criterion to stop the training ([xyz grouped, mean square error]), the mean distances between the interpolated and expected points vary from $0.51 \mathrm{~mm}$ to $3.94 \mathrm{~mm}$. For the configuration with one ANN for each coordinate and the same criterion to control the ANN training ([x/y/z separated, mean square error]), the mean distances between the interpolated and expected points vary from $0.70 \mathrm{~mm}$ to $7.90 \mathrm{~mm}$. This configuration gives a better result for only one case: $h_{t}=1680.3 \mathrm{~mm}$; for all the others, the results are worse. The last configuration with $3 \mathrm{ANN}$ and the median as criterion to stop the training ([x/y/z separated, median]), the mean distances are the highest of the 3 configurations for $h_{t} \in\{1675.4 ; 1680.3\}$, and lower than the second one, but higher than the first in all the other cases. Thus 6 times out of 7, the mean error obtained with the [xyz grouped, mean square error] configuration is inferior to the others. Indeed, the first configuration interpolates the coordinates with the lowest error, which is almost always inferior to $1.93 \mathrm{~mm}$ and at most equal to $3.94 \mathrm{~mm}$. We assume that considering the 3 coordinates together is better than considering them separately since there is interaction among them. When they are considered separately, these interactions are not taken into account.

In addition, even if the results obtained with the use of the median are better than those found with the mean-square for the 5 ANN inputs, it is still less accurate than the mean-square with 9 inputs. This is probably due to the great number of coordinates to learn (26723). 


\subsubsection{Performance of the adaptation strategy for 3D lung contours}

The final tests were performed with the entire learning set and 3 new sets of 3D lung contours. For these 3 new sets, a height has been carefully chosen to test all the possible cases: the first height is just above the smaller stored one, the second is just bellow than the higher stored one, and the last is in the middle of the stored panel of stored heights. Only the 3D lung contours have been manually created by the experts, no other organ contours. Since the 3D lung contours alone were designed, these phantoms were used only for these last tests and not stored in the EquiVox case, nor where they used during the ANN learning. In addition, the same manual creation process has been followed for all of $L S$.

Table 5 shows the mean distances observed between the interpolated and expected points for each $h_{t}$ and ANN configuration. For the [xyz grouped, mean square error] configuration, the mean distances vary from $0.55 \mathrm{~mm}$ to $1.65 \mathrm{~mm}$. These distances are all inferior to the mean distances obtained with the third configuration ([x/y/z separated, median]) which vary between $2.17 \mathrm{~mm}$ and $5.84 \mathrm{~mm}$. This third configuration almost gives almost better results than the second ([x/y/z separated, mean square error]) for which the mean distances vary from $2.54 \mathrm{~mm}$ to $7.22 \mathrm{~mm}$. Therefore, the results presented in Table 5 show the superiority of the configuration using the mean-square error while the coordinates are learned together: the errors, which are equal or below $1.65 \mathrm{~mm}$, are the lowest. For the other configurations, the difference is slightly higher but still reasonable.

The calculations of dosimetric reports are usually computed using a voxelised phantom. The commonly used dimensions of the voxels are $1.8 \mathrm{~mm}$ by $1.8 \mathrm{~mm}$ by $4.8 \mathrm{~mm}[19,28]$. For the first configuration (a single ANN), the largest mean error is equal to $1.65 \mathrm{~mm}$ (inferior to the spatial resolution of commonly used phantoms). Thus, such 3D lung contours, generated by this ANN, can be used to establish dosimetric reports. The other configuration with identically trained ANNs, delivers coordinates that are insufficiently accurate.

The results presented in this paper are encouraging and lead to the creation of 3D lung contours matching those of a target case. Nevertheless, this adaptation strategy is based on phantoms that may contain errors in comparison with the expected lung contours of a real victim: these contours are already representations of reality with uncertainties. Thus, biases may have been introduced by one or more $L C_{i}$ incorrectly designed. The ANN implemented in the EquiVox adaptation phase for 3D lung contours may reduce the impact of these errors since an ANN is an interpolation tool, but further research is needed to verify the necessary accuracy of $L S$. Another point is the generalisation of this adaptation strategy to the contours of the other organs. In fact, for other organ volumes and contours, one or more other parameters and measurements must be taken into account in the adaptation process, such as weight, fat-muscle proportion, and/or wrist diameter.

\section{CONCLUSION}

The EquiVox platform was developed to be used in emergency situations where a rapid and reliable decision is required in order to choose the best 3D phantom to perform dosimetry calculation and establish a dosimetric report. The choice is made using the CBR approach based on the feedback from previous similar experiences. EquiVox helps the experts in choosing the most adapted 3D phantom by means of the computation of indices for similarity and confidence. The similarity index defines the equivalence between the target case and the source case, whereas the confidence index highlights the uncertainty in the similarity calculation. The tests performed on an average set of target cases gave an efficiency of $85 \%$ in the application case of in vivo female counting for pulmonary anthroporadiametry. This version also presents an efficient and customisable graphic interface allowing comparison of the target to the source cases. 
In this version, different adaptation strategies for the interpolation of 3D lung contours were evaluated. The configuration based on a perceptron with one hidden layer, 9 inputs, 3 outputs, and a quasiNewton BFGS backpropagation-based method as an ANN training algorithm gave the best results. The different neural networks that were implemented produced convenient preliminary results showing that such an ANN can efficiently and quickly reproduce the exact positioning of different points once the learning step is achieved.

Further work will focus on two major axes. The first will be the evaluation of the biases inferred by each source case solution. Indeed, these solutions are phantoms designed manually by the experts and do not represent exact reality. Our ambition with this first axis is to propose a tool capable of determining the best learning subset for the ANN. The second is to enlarge this adaptation tool to include all other 3D organ contours of the phantoms. Hence, if the lung volumes and contours depend on a person's entire height, then other organ volumes and contours depend on one or more other measurements.

\section{ACKNOWLEDGEMENTS}

The authors want to acknowledge the SFRP (Société Française de Radioprotection), the LCC (Ligue Contre le Cancer), the PMA (Pays de Montbéliard Agglomération) for their financial aid, John Olsen and Ana-Maria Roxin for the translation corrections, and Emmanuelle Fontaine, Hamza Bouhelal, Maurice Bopp, Thomas Minot, and Julien Beurrier for their programming assistance.

\section{REFERENCES}

1. Broggio D, Zhang B, de Carlan L, Desbrée A, Lamart S, le Guen B, Bailloeuil C, Franck D., Analytical and Monte Carlo assessment of activity and local dose after a wound contamination by activation products, Health Phys., 96: 155-163; 2009.

2. Clairand I, Huet C, Trompier F, Bottollier-Depois J.-F., Physical dosimetric reconstruction of a radiological accident due to gammagraphy equipment that occurred in Dakar and Abidjan in summer 2006, Radiation Measurements, 43: 698-703; 2008.

3. De Carlan L., Aubineau-Lanièce I., Lemosquet A., Borissov N., Jourdain J.R., Jeanbourquin D., Le Guen B., Franck D., Application of new imaging and calculation techniques to activity and dose assessment in the case of a 106Ru contaminated wound, Radiat. Prot. Dosim., 105: 219-223; 2003.

4. Huet C., Lemosquet A., Clairand I., Rioual J.B., Franck D., de Carlan L., Aubineau-Lanièce I., Bottollier-Depois J.F., SESAME: a software tool for the numerical dosimetric reconstruction of radiological accidents involving external sources and its application to the accident in Chile in December 2005, Health Phys., 96: 76-83; 2009.

5. Sauget M., Laurent R., Henriet J., Salomon M., Gschwind R., Contassot-Vivier S., Makovicka L., Soussen C., Efficient domain decomposition for a neural network learning algorithm used for the dose evaluation in external radiotherapy: $20^{\text {th }}$ International Conference on Artificial Neural Networks, LNCS, Springer, 6352: 261-266; 2010.

6. Kolodner J., Reconstructive Memory: A Computer Model, Cognitive Science 7: 4; 1983.

7. Henriet J, Farah J., Chebel-Morello B., Bopp M., Broggio D., Makovicka L., Feasability Study of a New Platform Based on the Case-Based Reasoning Principles to Efficiently Search and Store Voxel Phantoms, Radioprotection, 45(1): 67-82; 2010. 
8. Bopp M., Henriet J., Chebel-Morello B., Makovicka L., Broggio D., Preliminary Study of a New CBR-Based Application for Voxelised Phantom Creation: ReEPh, in Singaporean-French IPAL Symposium (SinFra'09): 107-115; February 18-20, 2009.

9. McCulloch W, Pitts W., A logical calculus of ideas immanent in nervous activity, Bulletin of Mathematical Biophysics, 5:115-133; 1943.

10. Laurent R., Henriet J., Salomon M., Sauget M., Gschwind R., Nguyen F., Makovicka L., Simulation of Lung Motion using an Artificial Neural Network, Cancer / Radiothérapie, Elsevier, in press, ref. Ms. No. CANRAD-D-10-00057R4; 2010.

11. Makovicka L., Vasseur A., Sauget M., Martin E., Gschwind R., Henriet J., Salomon M., The future of new calculation concepts in dosimetry based on the Monte Carlo methods, in Radioprotection, 44(1): 77-88; 2009.

12. ICRP89, Basic Anatomical and Physiological Data for Use in Radiological Protection. International Commission on Radiological Protection Publications 89; 2002.

13. ICRU, Phantoms and computational models in Therapy, Diagnosis and Protection. International Commission on Radiation Units and Measurements Report 48; 1992.

14. Kramer, R; Zankl, M; Williams, G; Dexter, G., The calculation of dose from external photon exposures using reference human phantoms and Monte Carlo methods. Part I: the male (Adam) and female (Eva) adult mathematical phantoms, in Report GSF-Bericht S-885; 1982.

15. Tanaka, G I; Kawamura, H; Nakahara, Y., Reference Japanese man-I. Mass of organs and other characteristics of normal Japanese, in Health Physics, vol. 36, issue 3, pp. 333-346; 1979.

16. $\mathrm{Xu}, \mathrm{X}$ G, Chao, $\mathrm{T} \mathrm{C}$ et Bozkurt, A., VIP MAN, an imaged-based wholebody adult male model constructed from color photographs of the visible human project for multi-particle Monte Carlo calculations. Health Physics, vol. 78, issue 5, pp. 476-486; 2000.

17. Zankl, M., Viet, R., Williams, G., Schneider, K., Fendel, H., Petoussi, N., Dexler, G., The construction of computer tomographic phantoms and their application in radiology and radiation protection. Radiation Environmental Biophysics, vol. 27, pp. 153-164; 1988.

18. Zubal, I G; Harrell, C R; Smith, E O; Rattner, Z; Gindi, G; Hoffer, P B., Computerized three dimensional segmented human anatomy. Medical Physics, vol. 21, issue 2, pp. 299-302; 1994.

19. Farah, J., Broggio, D. et Franck, D., Examples of Mech and NURBS phantoms to study the morphology effect over in vivo lung counting, in Radiation Protection and Dosimetry Special Issue, Vol. 144, pp. 344-348; 2011.

20. Christensen, G. E., Deformable shape models for anatomy. Washington University. PhD Thesis; 1994.

21. Clairand, I., Bouchet, L. G., Ricard, M., Durigon, M., Di Paola, M., Aubert, B., Improvment of internal dose calculations using mathematical models of different adult heights, in Phys. Med. Biol., Vol. 45, pp. 2771-2785; 2000.

22. McNeel, Rhinoceros Modeling tools for designers. http://www.rhino3d.com.

23. Dassault Systems, CATIA - Virtual Design for Product Excellence. http://www.3ds.com/products/catia/welcome. 
24. Hsieh, W., Learning Methods in the Environmental Sciences - Neural Networks and Kernels, in Cambridge University Press; 2009.

25. Vasseur, A., Makovicka, L., Martin, E., Sauget, M. Contassot-Vivier, S., Bahi, J., Dose calculations using artificial neural networks: A feasibility study for photon beams, in Nuclear Instruments and Methods in Physics Research Section B: Beam interactions with materials and atoms, Vol. 266, pp. 1085-1093; 2008.

26. (CEN), European Committee for Standardization, Size designation of clothes: -part 1. Terms definitions and body measurement procedure, EN 13402-1 (ISO 3635: 1981 modified); 2001.

27. Robinette, K., M. CAESAR. measures up, Ergonomics in Design 8(3) pp. 17-23. 2000.

28. Farah, J., Broggio, D. et Franck, D., Creation and use of adjustable 3D phantoms: application for the lung monitoring of nuclear workers. European Conference on Individual Monitoring of Ionizing Radiation. Athens, Greece : s.n., 2010.

29. Kramer, G. H. et Burns, L. C., Evaluation of the effect of chest wall thickness, tissue composition and photon energy on the quantity muscle equivalent chest-wall-thickness by Monte Carlo simulation, in Radiat. Prot. Dosim., Vol. 82, pp. 115-124; 1999.

30. Digiteo, Scilab Home Page. http://www.scilab.org. 2011.

31. Ponce, J., Brette, R., Polynomial interpolation. Introduction to scientific computing and its applications. http://audition.ens.fr/brette/calculscientifique/2006-2007/lecture2.pdf. 2010. 